\title{
Revisión y mejora de la caracterización del grado de impermeabilidad requerido por el CTE DB-HS1 para fachadas de edificación
}

\author{
Review and improvement of the water tightness degree required \\ by the CTE DB-HS1 for building façades
}

J.M. Pérez-Bella ${ }^{(*)}$, J. Domínguez-Hernández $^{(*)}$, B. Rodríguez-Soria ${ }^{(*)}$, J.J. del Coz-Díaz ${ }^{(* *)}$, E. Cano-Suñén ${ }^{(*)}$

\section{RESUMEN}

El Código Técnico de la Edificación aprobado en 2006, asigna por primera vez en España diferentes requisitos básicos para los cerramientos verticales de edificación, destinados a limitar el riesgo de presencia inadecuada de humedad en el interior de los edificios. La solicitación por humedad, estimada mediante diversos parámetros climáticos, permite ajustar la exigencia de estos requisitos para cada emplazamiento y fachada. Sin embargo, estos parámetros presentan actualmente posibilidades de mejora que pueden dar lugar a una asignación más adecuada del grado de impermeabilidad y con ello, de los requisitos de diseño necesarios. Este artículo revisa estos parámetros climáticos, presenta otros utilizados internacionalmente para el mismo propósito y caracteriza en el territorio español los factores que determinan la solicitación por humedad, haciendo posible mejorar la prestacionalidad de los requisitos básicos impuestos para las fachadas de los edificios.

Palabras clave: Lluvia batiente; presión eólica; estanqueidad; Código Técnico de la Edificación; diseño de fachadas.

\section{ABSTRACT}

The Spanish Technical Building Code published in 2006 regulates the basic requirements of building façades to prevent the inadequate presence of atmospheric water inside Spanish buildings. The moisture exposure estimate, based on several climate parameters, allows adjusting these requirements for every location and façade. However, the parameters used by the Spanish Code can be improved, resulting in a more appropriate estimate of the required water tightness degree and thus in an improvable allocations of the necessary design conditions. This paper reviews these parameters, presenting other internationally applied, and analyses the moisture exposure in the Spanish territory, which would allow improving the subsequent performance of the basic requirements imposed to the façades.

Keywords: Driving rain; wind pressure; water tightness; Spanish Technical Building Code (CTE); façade design.

\author{
(*) Universidad de Zaragoza (España). \\ (**) Universidad de Oviedo (España). \\ Persona de contacto/Corresponding author: javdom@unizar.es (J. Domínguez-Hernández)
}

Cómo citar este artículo/Citation: Pérez-Bella, J. M., Domínguez-Hernández, J., Rodríguez-Soria, B., del Coz-Díaz, J. J., Cano-
Suñén, E. (2015). Revisión y mejora de la caracterización del grado de impermeabilidad requerido por el CTE DB-HS1 para fachadas de
edificación. Informes de la Construcción, 67(537): eo59, doi: http://dx.doi.org/10.3989/ic.13.004. Licencia / License: Salvo indicación contraria, todos los contenidos de la edición electrónica de Informes de la Construcción se distribuyen bajo una licencia de uso y distribución Creative Commons Reconocimiento no Comercial 3.o. España (cc-by-nc). 


\section{INTRODUCCIÓN}

El humedecimiento y la penetración del agua atmosférica en los cerramientos de edificación reducen su durabilidad (1) (2), disminuyen la capacidad de aislamiento de los materiales incrementando los costes energéticos del edificio (3) (4) y pueden llegar a afectar a la salud de sus ocupantes (5) (6). Por todo ello el Código Técnico de la Edificación (CTE) aprobado en 2006 en España, recoge como una de sus exigencias básicas garantizar la salubridad de los edificios, estableciendo la necesidad de limitar el riesgo previsible de presencia inadecuada de agua o humedad en los mismos (7).

Para ello, el Documento Básico de Salubridad - Protección frente a la humedad (DB-HS1) del CTE, establece las condiciones de diseño que las fachadas han de cumplir en relación a la solicitación por humedad atmosférica estimada sobre ellas. La determinación adecuada de esta solicitación, es por tanto un parámetro clave para garantizar la prestación de la solución constructiva asignada y con ello optimizar su diseño.

El humedecimiento de los cerramientos verticales de edificación se debe fundamentalmente a la combinación simultánea de precipitaciones atmosféricas y de rachas de viento (8). La acción del viento desvía la trayectoria vertical natural de las gotas de agua durante las precipitaciones, siendo responsable del aporte de agua líquida sobre la superficie vertical de las fachadas, denominado lluvia batiente (wind-driven rain o $W D R$ ) en la literatura especializada. Además, la presión eólica simultánea a la lluvia batiente sobre la fachada (driving rain wind pressure o $D R W P$ ), contribuye decisivamente a la penetración de este aporte de agua hacia el interior del cerramiento, incrementando el riesgo de presencia de humedad en el interior del edificio (9).

Sin embargo, para estimar la solicitación por humedad prevista en las fachadas, el DB-HS1 se apoya en dos parámetros climáticos (velocidad básica del viento y pluviometría media anual), que no permiten cuantificar ni la cantidad de agua atmosférica recibida por los cerramientos verticales, ni la presión eólica simultánea a dicho aporte de humedad. El resultado obtenido puede así caracterizar inadecuadamente el grado de solicitación existente, provocando asignaciones de soluciones constructivas para las fachadas, que no se ajusten a la exigencia climática.

Este artículo revisa los parámetros considerados por el DBHS1 para caracterizar la solicitación por humedad, identifica diversos ámbitos de mejora y analiza los procedimientos utilizados internacionalmente para determinar la lluvia batiente y la presión eólica simultánea a la precipitación.

El análisis de ambos parámetros WDR y DRWP en 80 emplazamientos repartidos por todo el territorio español, puede ser utilizado para mejorar la actual caracterización del grado de impermeabilidad realizada por el DB-HS1. Esta nueva caracterización de la solicitación por humedad en fachadas, puede contribuir a mejorar la asignación de las condiciones de diseño necesarias y a desarrollar un cumplimiento más prestacional de la exigencia básica de protección frente a la humedad.

\section{PARÁMETROS CLIMÁTICOS CONSIDERADOS POR EL DB-HS1 PARA LA ESTIMACIÓN DE LA SOLICITACIÓN POR HUMEDAD}

El DB-HS1 asigna un grado de impermeabilidad mínimo para las fachadas, según la solicitación prevista por agua atmosférica (7). La determinación de esta solicitación se realiza mediante dos parámetros que relacionan las condiciones climáticas de cada emplazamiento con la prestación demandada para la fachada.

El primero de estos parámetros es el valor básico de velocidad del viento o valor de la velocidad de viento que registrado

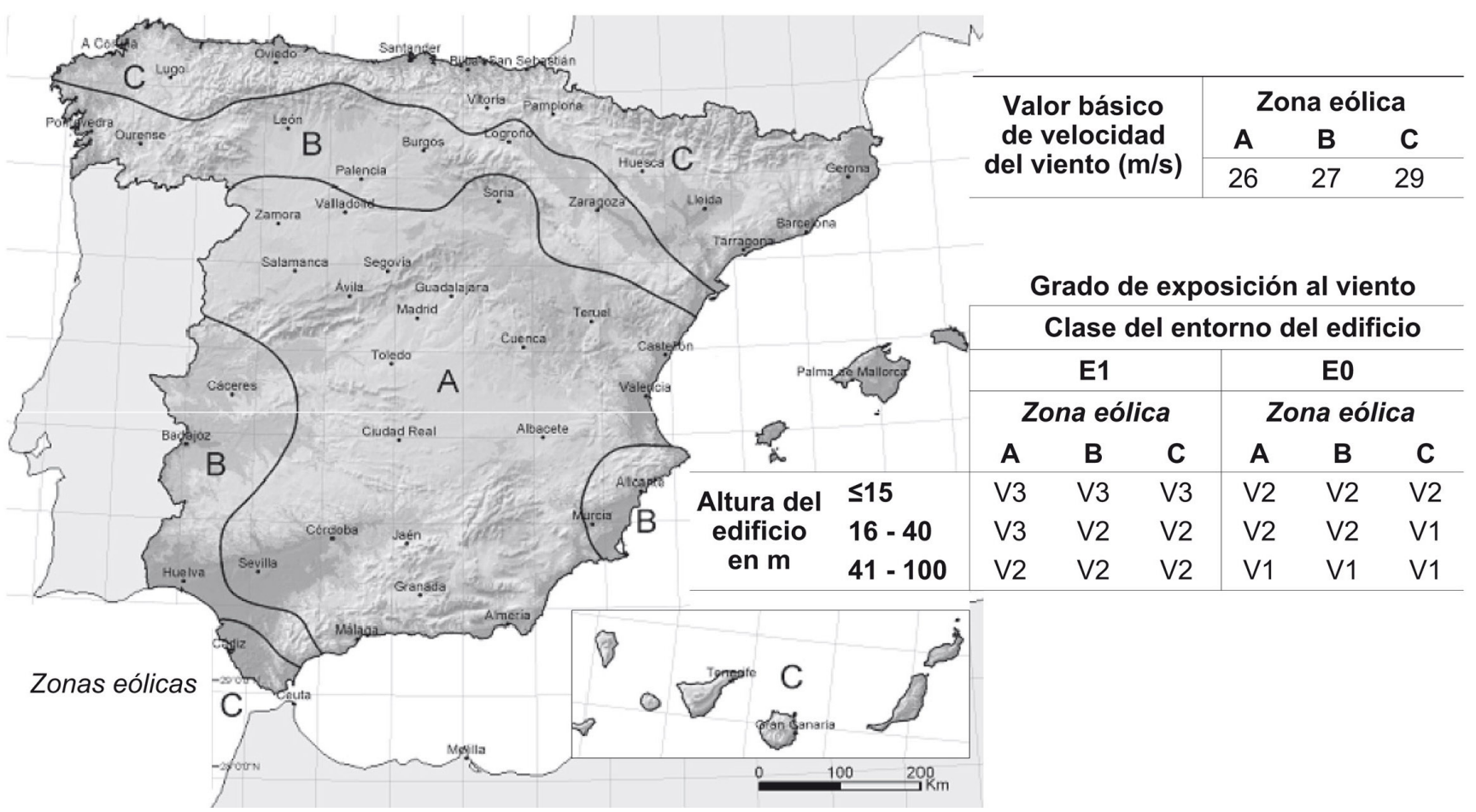

Figura 1. Zonas eólicas definidas en base al valor básico de la velocidad del viento y asignación del grado de exposición al viento. 
en un intervalo de 10 minutos, se espera que sea superado de media una vez cada 50 años (es decir, aquél que se repite con un periodo de retorno de 50 años). La combinación de una asignación zonal basada en este valor (zonas A, B o C), con la altura del edificio y con la clase de terreno en su entorno (Eo para zonas llanas y expuestas o E1 para zonas urbanas, industriales y forestales), determina el grado de exposición al viento (V1, V2 o V3) al que el cerramiento habrá de hacer frente (Figura 1).

El segundo de los parámetros se refiere a la pluviometría media anual recogida en el emplazamiento o valor de precipitación que se registra de media sobre una superficie horizontal a lo largo de un año. Una asignación zonal basada en este valor (zonas I, II, III, IV y V), junto con el anterior grado de exposición al viento, permite determinar el grado de impermeabilidad -1, 2, 3, 4 ó 5- requerido para la fachada (Figura 2).

Las condiciones de diseño requeridas por el DB-HS1 para cada fachada, se establecen según el grado de impermeabilidad obtenido, asignando diferentes soluciones constructivas que se consideran aceptables para hacer frente a cada solicitación. En consecuencia, la prestación de la solución constructiva depende directamente de la fiabilidad del grado de impermeabilidad y por lo tanto, de los parámetros utilizados para su determinación.

No obstante, diversos motivos impiden que el valor básico de velocidad del viento y la pluviometría media anual caractericen adecuadamente el grado de impermeabilidad necesario y con él, el riesgo existente de penetración de agua en el cerramiento:

1. Ambos parámetros no se refieren a intervalos temporales comparables. Mientras el valor de pluviometría se asocia a una exposición anual, la velocidad básica del viento hace referencia al valor máximo que se puede presentar durante un intervalo de tan solo 10 minutos de duración. Conse- cuentemente, ambos parámetros no pueden ser combinados para caracterizar una solicitación rigurosa, referida a un intervalo de tiempo común.

2. Tampoco ambos parámetros son conceptualmente relacionables, ya que mientras la pluviometría se refiere a un valor medio, promedio anual de registros meteorológicos pasados, la velocidad básica del viento se asocia al valor máximo estimado para un periodo de retorno de 50 años, valor por lo tanto obtenido mediante previsiones estadísticas de eventos máximos.

3. El valor de pluviometría, por sí solo no permite caracterizar la cantidad de agua que incide sobre las fachadas, ya que no valora la acción del viento simultánea a dichas precipitaciones, responsable de la desviación de las gotas de lluvia sobre los cerramientos verticales. Por lo tanto, la precipitación registrada sobre una superficie horizontal, no puede ser considerada una medida representativa del grado de humedecimiento de las fachadas.

4. El valor de velocidad básica del viento, tampoco es representativo de la presión eólica que se produce simultáneamente a los eventos de precipitación (ya que para su cálculo se analizan indistintamente registros de vientos simultáneos o no a la precipitación), por lo que este parámetro no es válido para caracterizar la influencia de la presión eólica en el proceso de penetración de agua en las fachadas.

5. El procedimiento utilizado para determinar el grado de exposición al viento, basado en un mapa zonificado y una asignación tabulada (Figura 1), no permite asociar un valor numérico a dicho grado de exposición. La ausencia de una caracterización cuantitativa, conlleva una inherente indeterminación que impide comparar con exactitud la exposición al viento en diferentes emplazamientos o condiciones de servicio del cerramiento.

Todo lo anterior contribuye a que el grado de impermeabilidad estimado para fachadas sea mejorable y en consecuencia, también lo sea la asignación de la solución constructiva nece-

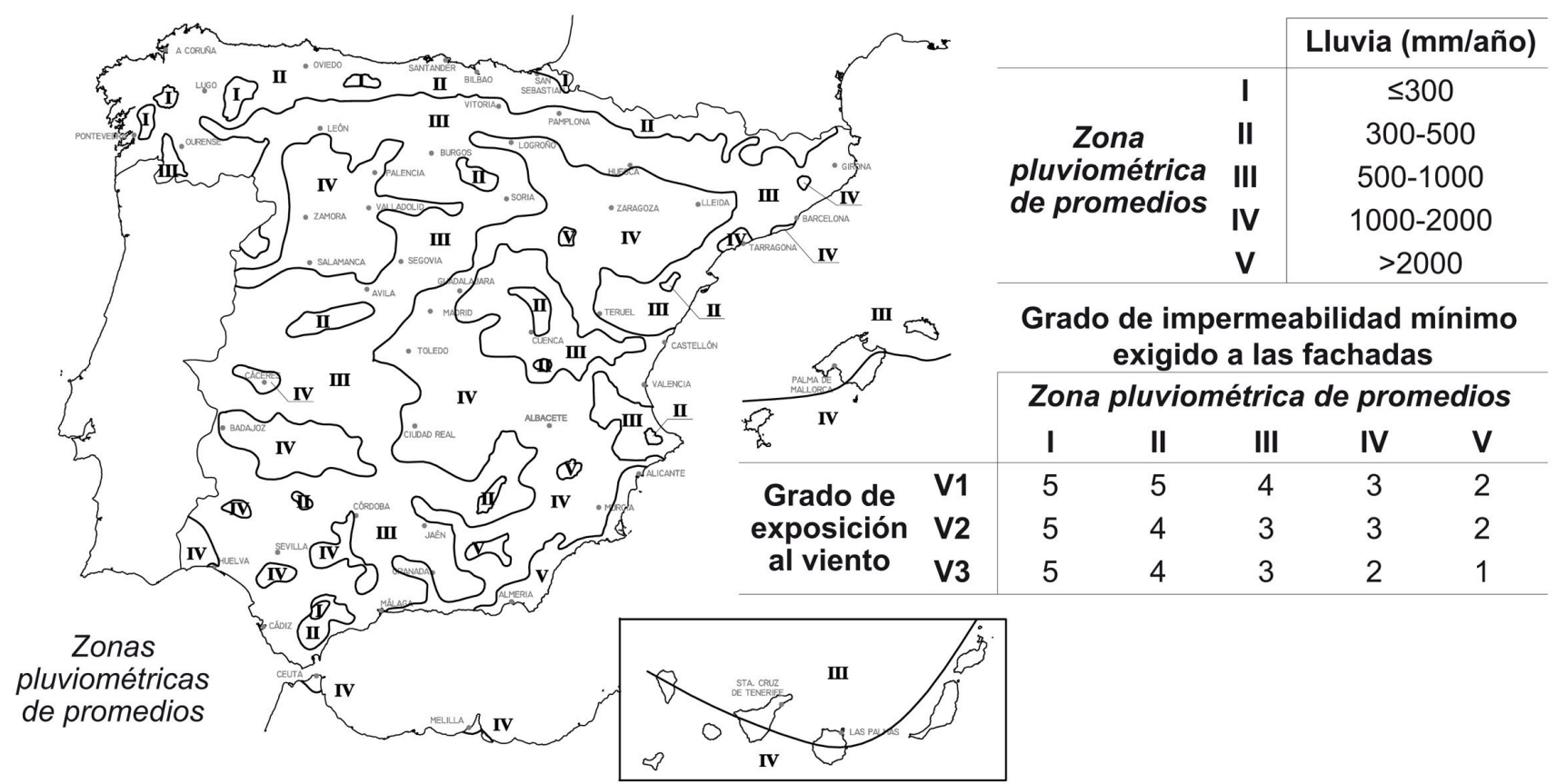

Figura 2. Zonas pluviométricas de promedios definidas en base al índice pluviométrico anual y asignación del grado de impermeabilidad mínimo requerido para las fachadas. 
saria. La revisión realizada en este trabajo de los parámetros climáticos considerados para caracterizar el grado de impermeabilidad, presenta nuevas posibilidades para dotar de una mayor prestacionalidad al DB-HS1.

\section{CARACTERIZACIÓN INTERNACIONAL DE LA SOLICITACIÓN POR HUMEDAD EN FACHADAS}

Para determinar con fiabilidad la solicitación por humedad sobre las fachadas de los edificios, es necesario cuantificar tanto el aporte de agua atmosférica recibido por las mismas (responsable del humedecimiento del cerramiento), como la presión eólica simultánea a la precipitación (responsable de su penetración al interior del mismo) (9). Para ello en la actualidad se dispone en el ámbito internacional de diferentes procedimientos de cálculo, que permiten determinar con precisión ambos parámetros de interés:

\subsection{Determinación del aporte de agua recibido por las fachadas}

Para caracterizar la exposición del cerramiento vertical al humedecimiento, desde los años 50 y 60 del siglo pasado, diversos investigadores europeos abordaron durante los eventos de precipitación la medición simultánea de la intensidad de lluvia, de la velocidad del viento y de la lluvia batiente recibida por las superficies verticales (10) (11).

El resultado de estos trabajos de campo permitió establecer una ecuación semi-empírica (conocida como relación WDR o WDR-relationship), capaz de estimar el valor de lluvia batiente que reciben las fachadas de cualquier emplazamiento, únicamente a partir del registro simultáneo de ambos datos meteorológicos (Figura 3). Así, el cálculo de la lluvia batiente $W D R\left(1 / \mathrm{m}^{2}\right)$ recibida, se aproxima como el producto de la velocidad del viento $U(\mathrm{~m} / \mathrm{s})$, de la intensidad de la precipitación registrada sobre una superficie horizontal $R_{h}\left(\mathrm{l} / \mathrm{m}^{2}\right)$ y de un coeficiente empírico de ajuste $k(\mathrm{~s} / \mathrm{m})[1]$.

$W D R=k \cdot U \cdot R_{h}$
La relación [1], aunque asume un flujo estacionario y horizontal para el viento, perpendicular a la superficie del cerramiento vertical analizado, constituye la base de todo el desarrollo posterior en este campo, ya que posibilita estimar la exposición al humedecimiento de cualquier fachada únicamente a partir de los datos climáticos de viento y lluvia registrados en el emplazamiento. La precisión de la estimación de $W D R$, depende tanto de la idoneidad de los datos meteorológicos empleados como del factor de ajuste $k$ adoptado.

Para una mayor precisión, el viento $U$ y la precipitación $R_{h}$ han de ser registrados simultáneamente en intervalos de tiempo de la menor duración posible, minimizando así los posibles errores debidos al promedio de los registros y a la no simultaneidad de ambos datos meteorológicos (12) (13).

Por su parte, el valor del coeficiente de ajuste $k$ depende del tamaño de la gota de agua característico de cada episodio de precipitación, siendo mayor cuanto mayor es la intensidad de estos episodios. Diferentes estudios de campo han propuesto valores medios para dicho coeficiente que habitualmente oscilan entre 0,20 y $0,25 \mathrm{~s} / \mathrm{m}$ (10) (14) (15) (16).

La aplicación más sencilla de esta relación WDR se alcanza omitiendo este coeficiente de ajuste $k$. Se obtiene así un índice de lluvia batiente $\left(\mathrm{m}^{2} / \mathrm{s}\right)$ o driving rain index (DRI), habitualmente promediado para un periodo anual ( $a D R I$ ), que permite comparar de forma aproximada el nivel de exposición a la humedad atmosférica entre diferentes emplazamientos. Para su cálculo, es frecuente utilizar valores promedio anuales de precipitación $R_{h}$ ( $\mathrm{mm} / \mathrm{año}$ ) y velocidad de viento $U(\mathrm{~m} / \mathrm{s})$ registrados a lo largo de $N$ años en cada emplazamiento, como muestra la ecuación [2] (17).

$$
a a D R I=\frac{\sum_{i=1}^{N} U \cdot\left(\frac{R_{h}}{1000}\right)}{N}
$$
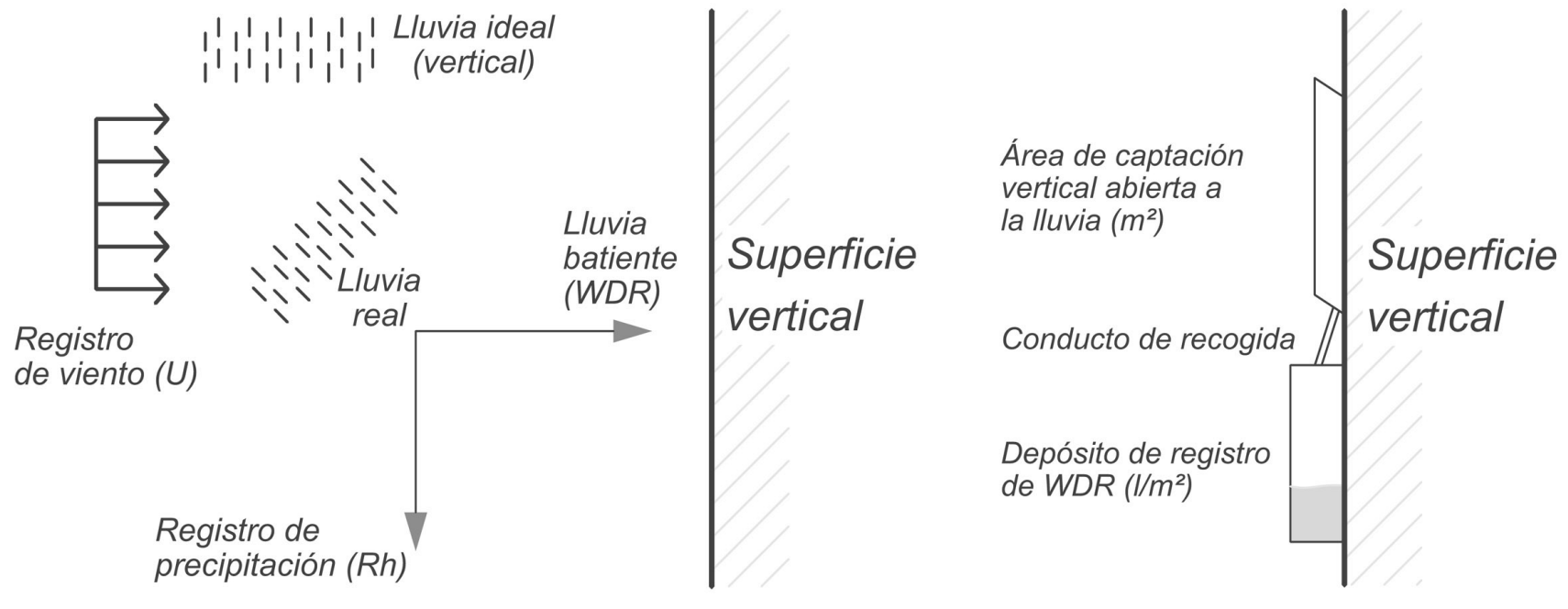

\section{Superficie horizontal}


El uso de registros meteorológicos referidos a intervalos menores (como el diario o mensual), permite obtener mayor precisión en la caracterización de la exposición, al reducir el error de promedio cometido y desechar la velocidad de viento de aquellos intervalos (diarios o mensuales) en los que no se produce precipitación alguna. En dichos casos, el índice se denomina daDRI o maDRI respectivamente.

A pesar de no cuantificar el volumen real de agua recibida sobre las fachadas, por su simplicidad, estos índices han sido utilizados en las últimas décadas para caracterizar la exposición en un gran número de países (8) (18) (19). Recientemente también ha sido presentada una completa caracterización del índice daDRI, en diversos emplazamientos españoles (20).

\subsubsection{El estándar internacional actual}

En la actualidad el estándar ISO 15927-3:2009 (21), traspuesto fielmente a la norma UNE-EN ISO 15927-3:2011 (22), homogeneíza el uso internacional de la relación WDR, posibilitando además un análisis direccional del aporte de agua recibido sobre cada posible orientación del cerramiento. Para ello se sirve de datos climáticos horarios de viento y precipitación, estimando el aporte de lluvia batiente tanto a largo plazo (caracterizando el estado de humedecimiento promedio al que se ve sometido anualmente cada cerramiento), como a corto plazo (caracterizando el humedecimiento máximo que puede acumularse sobre el cerramiento antes de que las pérdidas por evaporación disminuyan la humedad en la fachada). La determinación de la exposición en base a ambos intervalos temporales, es considerada por el estándar como suficiente para caracterizar completamente la exigencia sobre el cerramiento.

A largo plazo, la lluvia batiente media anual $I_{A \Theta}\left(1 / \mathrm{m}^{2} \mathrm{año}\right)$ que se espera que reciba anualmente una fachada de orientación $\Theta\left({ }^{\circ}\right)$, se cuantifica mediante la relación [3], donde $R_{h}\left(1 / \mathrm{m}^{2}\right)$ representa la intensidad de lluvia, $U_{10}(\mathrm{~m} / \mathrm{s})$ la velocidad media del viento a una altura de 10 metros sobre la rasante de un terreno despejado y $D\left(^{\circ}\right)$ la dirección media del viento, todo ello registrado simultáneamente cada hora en el emplazamiento. Como coeficiente de ajuste $k$, se emplea un valor genérico e igual a 2/9 (s/m).

$$
I_{A \Theta}=k \cdot \frac{\sum_{i=1}^{m} U_{10} \cdot\left(R_{h}\right)^{8 / 9} \cdot \cos (D-\Theta)}{N}
$$

En el sumatorio presente en la ecuación [3], solo se consideran los $m$ registros horarios contenidos dentro del periodo de $N$ años analizados, en los que la dirección del viento $D$ impulsa agua sobre la fachada de orientación $\Theta$. Como se desprende de la formulación, debe realizarse un cálculo independiente para cada posible orientación $\Theta$ del cerramiento, lo que permite caracterizar el aporte de agua sobre cada posible fachada de los edificios.

A corto plazo, la lluvia batiente se analiza únicamente a lo largo de cada periodo continuado de aporte de agua sobre la fachada. Para ello la norma introduce el concepto de periodo de humedecimiento, definiendo su duración como aquella limitada por 96 horas carentes de lluvia batiente sobre la fachada de orientación $\Theta$, momento a partir del cual se considera que las pérdidas de humedad por evaporación se hacen predominantes, concluyendo el intervalo de progresivo humedecimiento del cerramiento (Figura 4).

La máxima lluvia batiente $I_{S \Theta}\left(1 / \mathrm{m}^{2}\right.$ año) que se recibe sobre la fachada antes de que la misma comience a secarse, se determina mediante la relación [4], considerando en el sumatorio, únicamente los $m$ registros horarios contenidos dentro de cada uno de estos periodos de humedecimiento, en los que la dirección $D$ del viento impulsa una cantidad de agua no nula sobre la fachada de orientación $\Theta$. De nuevo, como coeficiente de ajuste $k$, se emplea un valor igual a $2 / 9(\mathrm{~s} / \mathrm{m})$.

$$
I_{S \Theta}^{\prime}=k \cdot \sum_{i=1}^{m} U_{10} \cdot\left(R_{h}\right)^{8 / 9} \cdot \cos (D-\Theta)
$$

Analizados todos los periodos de humedecimiento presentes a lo largo de la serie disponible de registros horarios, el valor de referencia a considerar $I_{S \Theta}\left(1 / \mathrm{m}^{2}\right.$ año), debe obtenerse determinando estadísticamente el valor máximo de lluvia batiente que puede producirse durante uno de ellos, para un periodo de retorno de 3 años (23).

Sin embargo, la utilización del estándar internacional se ve limitada en la mayoría de los países, incluyendo España, por la carencia de registros climáticos horarios recopilados en un número significativo de emplazamientos y con una antigüedad superior a 10 años (el estándar recomienda llegar a 20 ó 30 años de registros). Por ello, diversos investigadores han desarrollado procedimientos alternativos para cuantificar, de forma similar al estándar ISO, la exposición a la lluvia batiente mediante datos climáticos habitualmente disponibles (24).

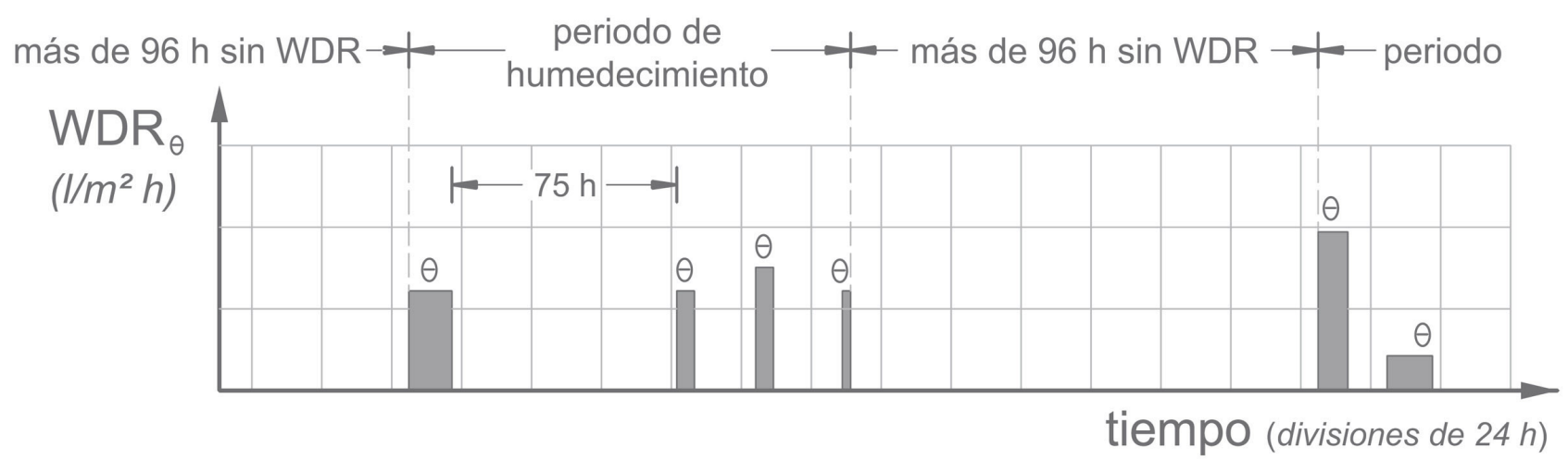

Figura 4. Definición de periodo de humedicimiento por el estándar ISO 15927-3:2009, para establecer el valor máximo de exposición puntual a la humedad sobre cada posible orientación $\Theta$ del cerramiento. 
Así, en el apartado 4 se presenta una adaptación de la formulación empleada por el estándar, que mediante el uso de registros diarios de viento y precipitación, permite disponer de un parámetro de solicitación capaz de sustituir al valor de pluviometría media actualmente utilizado por el DB-HS1.

\subsection{Determinación de la presión eólica simultánea a la lluvia batiente}

La presión eólica simultánea a la lluvia batiente es el segundo parámetro fundamental para la penetración de agua al interior del cerramiento. En consecuencia, todos los ensayos utilizados internacionalmente para evaluar la estanqueidad de las soluciones de fachada, consideran este parámetro como el conductor de la prueba (25) (26). Así, ante un aporte constante de agua, la máxima diferencia de presión que el cerramiento es capaz de soportar sin presencia de humedad en la cara interior del mismo, determina la prestación de estanqueidad de la solución constructiva.

La diferencia de presión o presión eólica simultánea a la precipitación $D R W P(\mathrm{~Pa})$ que se produce sobre una fachada de orientación $\Theta\left(^{\circ}\right)$, puede calcularse mediante la ecuación [5], donde $\rho_{\text {aire }}\left(\mathrm{kg} / \mathrm{m}^{3}\right)$ representa la densidad del aire, $U_{10}$ $(\mathrm{m} / \mathrm{s})$ la velocidad del viento y $D\left({ }^{\circ}\right)$ la dirección del viento, únicamente de los registros meteorológicos simultáneos a la lluvia.

$$
D R W P=\frac{1}{2} \cdot \rho_{\text {aire }} \cdot U_{10}^{2} \cdot \cos (D-\Theta)
$$

El valor medio anual $D R W P_{A \Theta}(\mathrm{Pa})$ característico de la exposición a largo plazo, debe calcularse como el promedio anual del valor de $D R W P$ en los años analizados, tal y como indica la ecuación [6]. Para ello, en el sumatorio solo se consideran aquellos $m$ registros simultáneos a la lluvia, en los que la dirección del viento genera una presión positiva sobre la fachada de orientación $\Theta$, propiciando la penetración del aporte de agua recibido. De nuevo, ha de realizarse un cálculo independiente para cada posible orientación $\Theta$ del cerramiento.

$$
D R W P_{A \Theta}=\frac{\sum_{i=1}^{m} \frac{1}{2} \cdot \rho_{\text {aire }} \cdot U_{10}^{2} \cdot \cos (D-\Theta)}{m}
$$

Análogamente, para determinar el valor de presión eólica asociado a cada periodo de humedecimiento (exposición a corto plazo), deben promediarse solo los valores de DRWP asociados a los registros incluidos dentro de cada uno de estos periodos. El valor de referencia para esta exposición $D R W P_{S \Theta}(\mathrm{Pa})$, se estima estadísticamente como el promedio máximo de presión eólica que puede producirse en uno de estos periodos, para un periodo de retorno de 3 años (23).

De nuevo, el uso de registros meteorológicos referidos a intervalos de corta duración, permitirá obtener estimaciones de $D R W P$ más precisas, al reducirse el error de promedio cometido y poder descartar aquellos registros de viento no simultáneos a la lluvia.

Actualmente pocos países han abordado la realización de estudios exhaustivos de DRWP en un número significativo de emplazamientos (27). Por ello, la solicitación por humedad en los cerramientos de los diferentes países se ha venido es- tableciendo únicamente mediante mapas de exposición a la lluvia batiente (28) (29), soslayando la relevancia que la presión eólica tiene en el riesgo de penetración de agua a través de la fachada.

No obstante, este procedimiento para determinar la $D R W P$ puede ser aplicado también en España, utilizando registros meteorológicos diarios y permitiendo así sustituir el parámetro de velocidad básica de viento que actualmente se emplea en el DB-HS1. La combinación de ambos parámetros WDR y $D R W P$ referidos a diferentes intervalos de exposición (a corto y largo plazo), es por lo tanto una labor ineludible para estimar con mayor precisión la exigencia de protección frente a la humedad necesaria en las fachadas españolas y en relación a la misma, regular las soluciones constructivas necesarias para cumplimentar dicho requisito.

\section{DETERMINACIÓN PRESTACIONAL DE LA SOLICITACIÓN POR HUMEDAD}

En España, la Agencia Estatal de Meteorología (AEMET) ha desarrollado tradicionalmente la labor de registro y recopilación de datos meteorológicos simultáneos de precipitación y velocidad de viento, si bien en intervalos mayores a los horarios exigidos por el estándar ISO. En consecuencia, en la actualidad no se dispone de datos horarios de antigüedad superior a 10, 20 ó 30 años en un número suficientemente representativo de estaciones meteorológicas.

Sin embargo, AEMET cuenta con otros registros meteorológicos, que pueden ser igualmente utilizados por procedimientos alternativos de cálculo para estimar tanto la lluvia batiente como la presión eólica simultánea a la misma en un elevado número de emplazamientos. Así, utilizando datos diarios registrados durante más de 30 años en 80 estaciones meteorológicas repartidas por todo el país, recientes estudios han permitido caracterizar el valor de WDR y DRWP asociado a periodos de humedecimiento, obteniendo con ello sendos mapas de exposición a corto plazo (20) (30).

Para ello fue empleada la misma formulación del estándar ISO, utilizando registros diarios en lugar de los datos horarios inicialmente requeridos por el estándar. Dado que estos registros diarios no ofrecen información acerca de la dirección del viento (variable a lo largo de un día completo), el cálculo soslayó el componente direccional de las ecuaciones, obteniendo así un resultado escalar. Igualmente, para el cálculo de la presión eólica simultánea a la lluvia batiente, estos estudios se sirvieron de la expresión [5], eliminando también el componente direccional de la misma.

La caracterización así obtenida determinó la exposición global a $W D R$ y $D R W P$ sobre el conjunto de todos los cerramientos verticales en el emplazamiento, independientemente de su orientación específica. Estos resultados escalares demostraron ser convergentes con los resultados direccionales obtenidos mediante el propio estándar ISO (20), posibilitando así cuantificar a lo largo de un periodo de humedecimiento, la cantidad de agua atmosférica recibida y la presión eólica simultánea, en las fachadas de cada uno de los 80 emplazamientos españoles analizados.

Sin embargo, esta determinación del valor de $W D R$ y $D R W P$ que puede acumularse sobre un cerramiento durante un periodo de humedecimiento, necesita también ser complemen- 
tada con el valor de exposición promedio sobre dichos cerramientos (exposición a largo plazo), si se pretende caracterizar completamente la solicitación por humedad.

\subsection{Cálculo de la exposición a largo plazo de WDR y $D R W P$ en España}

Como punto de partida para el cálculo medio anual de ambos parámetros, se han empleado las series de registros meteorológicos diarios facilitados por AEMET, correspondientes a las mismas 80 estaciones meteorológicas analizadas en los estudios precedentes. Estas series cuentan siempre con más de 30 años de registros, alcanzando 50 años (1962-2011) en 35 de los emplazamientos y ofrecen información diaria relativa a la precipitación registrada, velocidad media del viento, temperatura media y rangos de presión atmosférica máxima y mínima.

Para calcular el aporte de agua recibido anualmente por las fachadas, se parte de la formulación del estándar ISO 159273:2009, aplicando las mismas adaptaciones utilizadas por estudios previos para determinar la exposición asociada a un periodo de humedecimiento (se elimina el componente direccional de la ecuación [3] y se utilizan datos diarios en lugar de horarios). Así, el valor escalar de lluvia batiente media anual $I_{A}\left(\mathrm{l} / \mathrm{m}^{2}\right)$ se calcula promediando en el número de años $N$ ana- lizados, el sumatorio del producto de todos los $m$ registros diarios disponibles de velocidad del viento $U_{10}(\mathrm{~m} / \mathrm{s})$ y precipitación $R_{h}\left(\mathrm{l} / \mathrm{m}^{2}\right)$, como muestra la ecuación [7]:

$$
I_{A}=\frac{2}{9} \frac{\sum_{i=1}^{m} U_{10} \cdot\left(R_{h}\right)^{8 / 9}}{N}
$$

El valor escalar obtenido, determina la cantidad anual de agua que se espera que reciban en un determinado emplazamiento, todas las fachadas de un edificio, caracterizando a largo plazo su exposición frente al humedecimiento. Los resultados de este análisis se representan geográficamente en la Figura 5 para los 80 emplazamientos analizados.

De forma análoga, para caracterizar la presión eólica media simultánea a la lluvia batiente $D R W P_{A}(\mathrm{~Pa})$, la ecuación [8] promedia para los $N$ años de registros diarios analizados, el sumatorio del producto de velocidad del viento $U_{10}(\mathrm{~m} / \mathrm{s})$ y de densidad del aire $\rho_{\text {aire }}\left(\mathrm{kg} / \mathrm{m}^{3}\right)$, de cada uno de los $m$ días con precipitación.

$$
D R W P_{A}=\frac{\sum_{i=1}^{m} \frac{1}{2} \cdot \rho_{\text {aire }} \cdot U_{10}{ }^{2}}{N}
$$

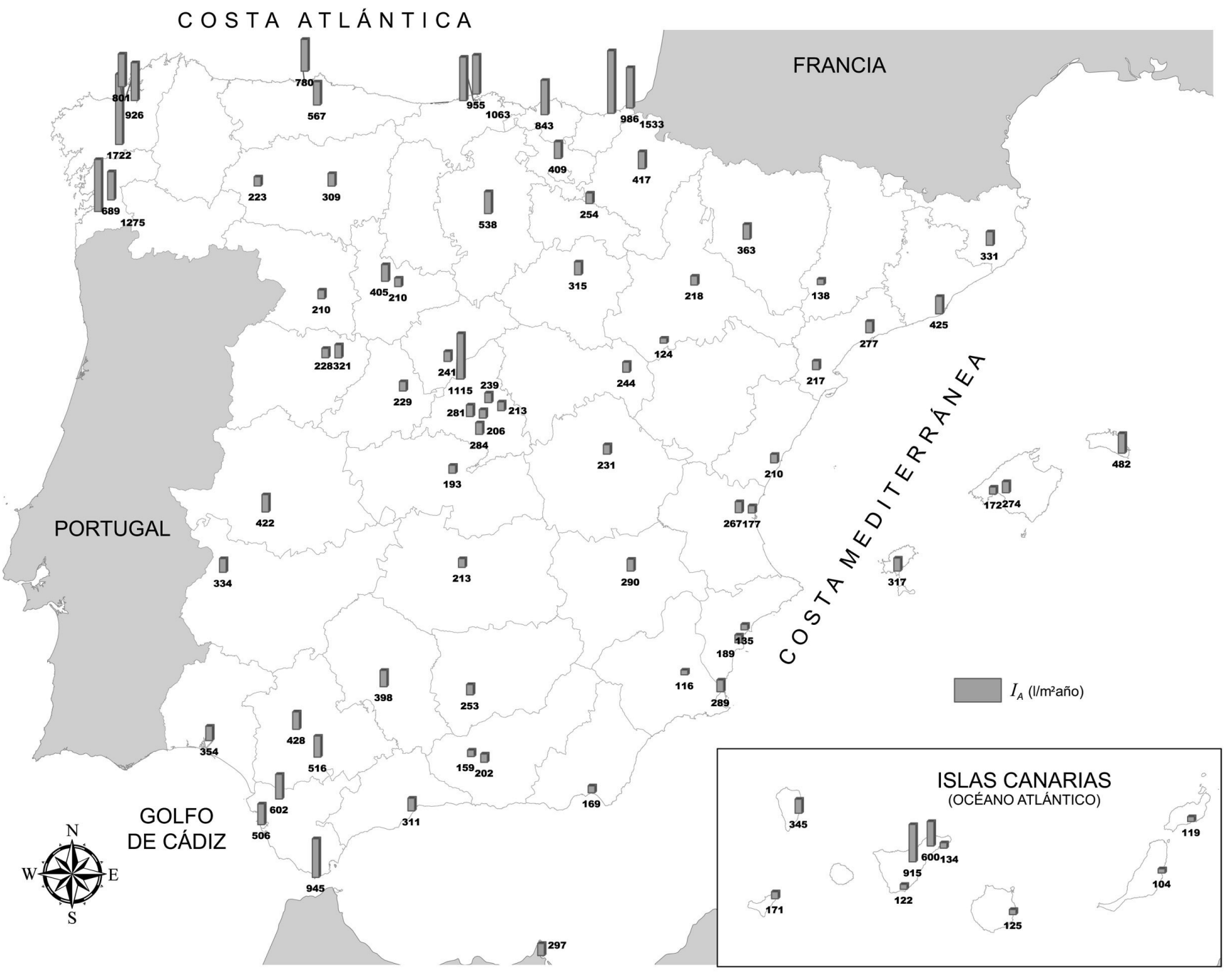

Figura 5. Mapa de exposición a la lluvia batiente referida a un intervalo anual. 
$\mathrm{El}$ aire se considera un gas ideal, determinando su densidad $\rho_{\text {aire } i}$ mediante la aproximación [9], donde $P_{i}$ es la presión absoluta $(\mathrm{Pa})$ obtenida como la media de las presiones máximas y mínimas registradas diariamente $\left(P_{\max i}\right.$ y $\left.P_{\min i}\right), T_{i}$ es la temperatura media diaria $(\mathrm{K})$ y $R$ es la constante universal de los gases ideales para el aire seco $(287,07 \mathrm{~J} / \mathrm{kgK})$.

$$
\rho_{\text {aire }}=\frac{P_{i}}{R \cdot T_{i}}=\frac{0,5\left(P_{\max i}+P_{\min i}\right)}{208,07 \cdot T_{i}}
$$

Los resultados escalares de presión, representan la presión eólica media simultánea al aporte de agua sobre el conjunto de todas las fachadas de un edificio, en cada emplazamiento. Estos resultados se representan geográficamente en la Figura 6, para los mismos 80 emplazamientos españoles anteriores.

Finalmente, los valores asociados a los índices de exposición a largo plazo $W D R_{A}$ y de $D R W P_{A}$, se desglosan en la Tabla 1, facilitando su posible utilización para una determinación mejorada del grado de impermeabilidad necesario en fachadas.

\subsection{Análisis de los resultados obtenidos}

Como se aprecia en la Figura 5, la exposición al humedecimiento de las fachadas es especialmente intensa en la costa atlántica del norte de la Península Ibérica y en áreas cercanas al Golfo de Cádiz, llegando a impactar una media de 1.720 l/ $\mathrm{m}^{2}$ año de agua sobre las fachadas en Santiago de Compostela. En el interior del país, la costa mediterránea y ambos archipiélagos, la lluvia batiente es sensiblemente inferior, aunque también pueden identificarse emplazamientos concretos en los que es comparable al de las zonas más solicitadas (Tenerife Norte aeropuerto $600 \mathrm{l} / \mathrm{m}^{2}$ año; Burgos $538 \mathrm{l} /$ $\mathrm{m}^{2}$ año; Menorca $482 \mathrm{l} / \mathrm{m}^{2}$ año; Barcelona $425 \mathrm{l} / \mathrm{m}^{2}$ año; Cáceres $422 \mathrm{l} / \mathrm{m}^{2}$ año y Villanubla $405 \mathrm{l} / \mathrm{m}^{2}$ año). En localizaciones de montaña como la Estación de Navacerrada o de Izaña, la solicitación es también elevada (en torno a $1.000 \mathrm{l} / \mathrm{m}^{2}$ año), si bien ambos datos no son representativos de las condiciones de exposición en núcleos urbanos de su entorno, ubicados a una altitud menor.

Analizando la Figura 6, se aprecia que este aporte de agua anual se combina con presiones eólicas simultáneas especialmente fuertes en zonas de la costa norte de la Península Ibérica, las Islas Canarias y puntos concretos del interior peninsular (Albacete 20 Pa; Burgos, Villanubla y Morón de la Frontera $19 \mathrm{~Pa}$ ) y de la costa mediterránea (Menorca $19 \mathrm{~Pa}$ ). Destaca la elevada presión eólica simultánea a la lluvia batiente identificada en emplazamientos como Tarifa (55 Pa) o Almería (18 Pa), que incrementa el riesgo de penetración del agua en los cerramientos, aun a pesar de la baja pluviometría.

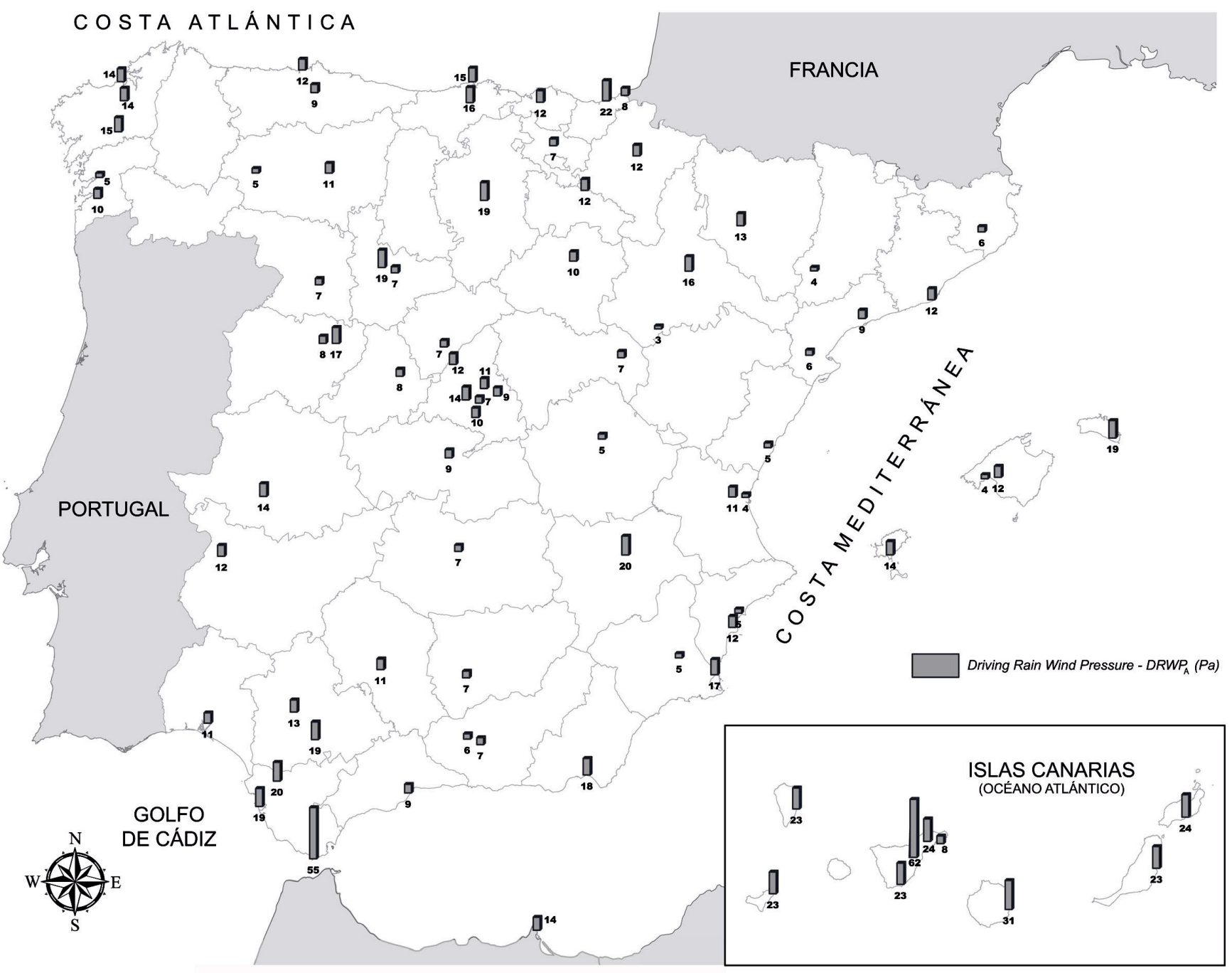

Figura 6. Mapa de exposición media a la presión eólica simultánea a la lluvia batiente. 
Tabla 1. Parámetros de solicitación obtenidos en los 80 emplazamientos analizados.

\begin{tabular}{|c|c|c|c|c|c|}
\hline EMPLAZAMIENTO & $\begin{array}{l}\text { Altitud } \\
\text { (m) }\end{array}$ & $\begin{array}{l}\text { Latitud } \\
\text { (DMS) }\end{array}$ & $\begin{array}{c}\text { Longitud } \\
\text { (DMS) }\end{array}$ & $\begin{array}{c}I_{A} \\
\left(1 / \mathbf{m}^{2} \mathbf{a n ̃ o}\right)\end{array}$ & $\begin{array}{l}\operatorname{DRWP}_{A} \\
\quad(\mathrm{~Pa})\end{array}$ \\
\hline La Coruña (aeropuerto) & 98 & $43^{\circ} 18 ’ 25 ” \mathrm{~N}$ & $08^{\circ} 22^{\prime} 20^{\prime \prime W}$ & 925,52 & 13,67 \\
\hline La Coruña & 58 & $43^{\circ} 22$ 'O2”N & $08^{\circ} 25^{\prime} 10^{\prime \prime} \mathrm{W}$ & 801,15 & 13,83 \\
\hline Santiago (aeropuerto) & 370 & $42^{\circ} 53 \prime 16 ” \mathrm{~N}$ & $08^{\circ} 24^{\prime} 39^{\prime \prime} \mathrm{W}$ & 1721,50 & 15,14 \\
\hline Vitoria (aeropuerto) & 513 & $42^{\circ} 52^{\prime} 55^{\prime} \mathrm{N}$ & $02^{\circ} 44^{\prime} 06^{\prime \prime W}$ & 409,05 & 7,27 \\
\hline Albacete (Los Llanos) & 704 & $38^{\circ} 57^{\prime} \mathrm{O} 8^{\prime \prime} \mathrm{N}$ & $01^{\circ} 51^{\prime} 47^{\prime \prime} \mathrm{W}$ & 290,46 & 20,38 \\
\hline Alicante (aeropuerto) & 43 & $38^{\circ} 16^{\prime} 58^{\prime \prime} \mathrm{N}$ & $\mathrm{Oo}^{\circ} 34^{\prime} 15 ” \mathrm{~W}$ & 189,36 & 11,95 \\
\hline Alicante & 81 & $38^{\circ} 22^{\prime} 21^{\prime \prime} \mathrm{N}$ & $\mathrm{Oo}^{\circ} 299^{\prime} 39^{\prime \prime} \mathrm{W}$ & 134,68 & 4,68 \\
\hline Almería (aeropuerto) & 21 & $36^{\circ} 50^{\prime} 47^{\prime \prime} \mathrm{N}$ & $\mathrm{O} 2^{\circ} 21^{\prime} 25^{\prime \prime} \mathrm{W}$ & 168,77 & 17,79 \\
\hline Asturias (aeropuerto) & 127 & $43^{\circ} 34^{\prime} \mathrm{O} 1 ” \mathrm{~N}$ & $06^{\circ} \mathrm{O} 2^{\prime} 39^{\prime \prime} \mathrm{W}$ & 779,56 & 11,63 \\
\hline Oviedo & 336 & $43^{\circ} 21^{\prime} 16^{\prime \prime} \mathrm{N}$ & $05^{\circ} 52^{\prime} 22 ” \mathrm{~W}$ & 567,09 & 8,81 \\
\hline Ávila & 1130 & $40^{\circ} 39^{\prime} 33^{\prime \prime} \mathrm{N}$ & $04^{\circ} 40^{\prime} 48^{\prime \prime} \mathrm{W}$ & 228,82 & 7,74 \\
\hline Badajoz (aeropuerto) & 185 & $38^{\circ} 53^{\prime}{ }^{\prime \prime} \mathrm{N}$ & $06^{\circ} 49^{\prime} 45^{\prime \prime} \mathrm{W}$ & 333,95 & 11,75 \\
\hline Barcelona (aeropuerto) & 4 & $41^{\circ} 17^{\prime} 34^{\prime \prime} \mathrm{N}$ & $02^{\circ} \mathrm{O} 4^{\prime} 12^{\prime} \mathrm{E}$ & 425,05 & 12,10 \\
\hline Burgos (Villafría) & 890 & $42^{\circ} 21^{\prime} 22 ” \mathrm{~N}$ & $03^{\circ} 37^{\prime} 57^{\prime \prime} \mathrm{W}$ & 538,42 & 19,15 \\
\hline Cáceres & 405 & $39^{\circ} 28^{\prime} 20^{\prime \prime} \mathrm{N}$ & $06^{\circ} 20^{\prime} 22 ” \mathrm{~W}$ & 422,33 & 13,75 \\
\hline Cádiz & 1 & $36^{\circ} 30^{\prime} 04^{\prime \prime} \mathrm{N}$ & $06^{\circ} 15^{\prime} 24^{\prime \prime W}$ & 506,00 & 19,34 \\
\hline Jerez (aeropuerto) & 27 & $36^{\circ} 45^{\prime} \mathrm{O} 2 ” \mathrm{~N}$ & $06^{\circ} 03^{\prime} 21^{\prime \prime} \mathrm{W}$ & 602,18 & 20,05 \\
\hline Tarifa & 32 & $36^{\circ} \mathrm{Oo}{ }^{\prime} 55^{\prime} \mathrm{N}$ & $05^{\circ} 35^{\prime} 51^{\prime \prime} \mathrm{W}$ & 944,65 & 55,00 \\
\hline Santander (aeropuerto) & 5 & $43^{\circ} 25^{\prime} 45^{\prime \prime} \mathrm{N}$ & $03^{\circ} 49$ '53”W & 1063,12 & 16,36 \\
\hline Santander & 52 & $43^{\circ} 29 ’ 30 ” \mathrm{~N}$ & $03^{\circ} 47^{\prime} 59^{\prime \prime W}$ & 954,88 & 14,77 \\
\hline Castellón de la Plana & 35 & $39^{\circ} 57^{\prime} 0{ }^{\prime \prime N} \mathrm{~N}$ & $\mathrm{OO}^{\circ} \mathrm{O} 4^{\prime} 17^{\prime \prime W}$ & 210,10 & 5,18 \\
\hline Ciudad Real & 628 & $38^{\circ} 59^{\prime} 22 ” \mathrm{~N}$ & $\mathrm{O} 3{ }^{\circ} 55^{\prime} 11^{\prime \prime} \mathrm{W}$ & 213,06 & 6,70 \\
\hline Córdoba (aeropuerto) & 90 & $37^{\circ} 50^{\prime} 39^{\prime \prime} \mathrm{N}$ & $04^{\circ} 50^{\prime} 46^{\prime \prime} \mathrm{W}$ & 398,34 & 11,47 \\
\hline Cuenca & 945 & $40^{\circ} \mathrm{O} 4^{\prime} \mathrm{OO} ” \mathrm{~N}$ & $02^{\circ} 08^{\prime} 17^{\prime \prime} \mathrm{W}$ & 231,40 & 5,39 \\
\hline Gerona (aeropuerto) & 143 & $41^{\circ} 54^{\prime} 42^{\prime \prime} \mathrm{N}$ & $\mathrm{O} 2^{\circ} 45^{\prime} 48^{\prime \prime} \mathrm{E}$ & 330,91 & 5,77 \\
\hline Granada (aeropuerto) & 567 & $37^{\circ} 11^{\prime} 23 ” \mathrm{~N}$ & $03^{\circ} 47^{\prime} 22 ” \mathrm{~W}$ & 159,33 & 5,69 \\
\hline Granada (base aérea) & 687 & $37^{\circ} 08^{\prime} 13 ” \mathrm{~N}$ & $03^{\circ} 37^{\prime} 53^{\prime \prime W}$ & 201,95 & 7,46 \\
\hline Molina de Aragón & 1056 & $40^{\circ} 50^{\prime} 40^{\prime \prime} \mathrm{N}$ & $\mathrm{O}^{\circ} 53^{\prime} \mathrm{O} 77^{\prime \prime W}$ & 243,54 & 6,90 \\
\hline San Sebastián (aeropuerto) & 4 & $43^{\circ} 21^{\prime} 25 ” \mathrm{~N}$ & $01^{\circ} 47^{\prime} 32^{\prime \prime W}$ & 986,20 & 7,90 \\
\hline San Sebastián (Igueldo) & 251 & $43^{\circ} 18 ’ 27^{\prime \prime} \mathrm{N}$ & $02^{\circ} 02^{\prime} 22^{\prime \prime} \mathrm{W}$ & 1533,02 & 21,84 \\
\hline Huelva & 19 & $37^{\circ} 16^{\prime} 48^{\prime \prime} \mathrm{N}$ & $06^{\circ} 54^{\prime} 35^{\prime \prime W}$ & 354,00 & 11,10 \\
\hline Huesca (aeropuerto) & 541 & $42^{\circ} \mathrm{O} 5{ }^{\prime} \mathrm{Oo} " \mathrm{~N}$ & $\mathrm{Oo}^{\circ} 19{ }^{\prime} 35^{\prime \prime W}$ & 363,30 & 13,16 \\
\hline Ibiza (aeropuerto) & 6 & $38^{\circ} 52^{\prime} 35^{\prime \prime} \mathrm{N}$ & $\mathrm{O} 1^{\circ} 23{ }^{\prime} \mathrm{O} 4^{\prime \prime} \mathrm{E}$ & 317,17 & 14,46 \\
\hline Menorca (aeropuerto) & 91 & $39^{\circ} 51^{\prime} 17^{\prime \prime} \mathrm{N}$ & $\mathrm{O} 4^{\circ} 12^{\prime} 56^{\prime \prime} \mathrm{E}$ & 482,05 & 18,80 \\
\hline Mallorca (aeropuerto) & 8 & $39^{\circ} 33399^{\prime \prime} \mathrm{N}$ & $\mathrm{O} 2^{\circ} 44^{\prime} 12^{\prime \prime} \mathrm{E}$ & 273,87 & 11,97 \\
\hline Palma de Mallorca (puerto) & 3 & $39^{\circ} 33^{\prime} 12^{\prime \prime} \mathrm{N}$ & $\mathrm{O} 2^{\circ} 37^{\prime} 31^{\prime \prime} \mathrm{E}$ & 172,15 & 4,42 \\
\hline Jaén & 582 & $37^{\circ} 46^{\prime} 40^{\prime \prime} \mathrm{N}$ & $03^{\circ} 48^{\prime} 27^{\prime \prime W}$ & 252,84 & 7,15 \\
\hline Logroño (aeropuerto) & 353 & $42^{\circ} 27^{\prime} \mathrm{O} 8^{\prime \prime} \mathrm{N}$ & $02^{\circ} 19{ }^{\prime} 52^{\prime \prime W}$ & 253,85 & 11,97 \\
\hline Fuerteventura (aeropuerto) & 25 & $28^{\circ} 26^{\prime} 41^{\prime \prime} \mathrm{N}$ & $13^{\circ} 51^{\prime} 47^{\prime \prime} \mathrm{W}$ & 103,86 & 23,37 \\
\hline Lanzarote (aeropuerto) & 14 & $28^{\circ} 57^{\prime} 07^{\prime \prime N}$ & $13^{\circ} 36^{\prime} 01 ” \mathrm{~W}$ & 118,81 & 24,19 \\
\hline Las Palmas (aeropuerto) & 24 & $27^{\circ} 55^{\prime} 21^{\prime \prime} \mathrm{N}$ & $15^{\circ} 23^{\prime} 22^{\prime \prime W}$ & 125,25 & 31,09 \\
\hline León (aeropuerto) & 916 & $42^{\circ} 35^{\prime} 20^{\prime \prime} \mathrm{N}$ & $05^{\circ} 38^{\prime} 58^{\prime \prime W}$ & 308,68 & 10,57 \\
\hline Ponferrada & 534 & $42^{\circ} 333^{\prime}=\mathrm{N}$ & $\mathrm{o6}^{\circ} 36^{\prime} \mathrm{Ooo"W}$ & 223,35 & 4,96 \\
\hline Lérida & 192 & $41^{\circ} 37^{\prime} 33 ” \mathrm{~N}$ & $\mathrm{OO}^{\circ} 35^{\prime} 42^{\prime \prime} \mathrm{E}$ & 137,57 & 3,98 \\
\hline Madrid (aeropuerto) & 609 & $40^{\circ} 28^{\prime} 00^{\prime \prime} \mathrm{N}$ & $03^{\circ} 33^{\prime} 20^{\prime \prime W}$ & 238,76 & 10,84 \\
\hline Madrid (Cuatro Vientos) & 687 & $40^{\circ} 22 ' 40^{\prime \prime} \mathrm{N}$ & $03^{\circ} 47^{\prime} 21 ” \mathrm{~W}$ & 281,25 & 13,94 \\
\hline Madrid (Getafe) & 617 & $40^{\circ} 18^{\prime} 0{ }^{\prime \prime} \mathrm{N}$ & $03^{\circ} 43^{\prime} 21$ ”W & 283,57 & 10,13 \\
\hline Madrid & 667 & $40^{\circ} 24^{\prime} 43^{\prime \prime} \mathrm{N}$ & $03^{\circ} 40^{\prime} 41^{\prime \prime} \mathrm{W}$ & 205,67 & 6,96 \\
\hline Madrid (Torrejón) & 611 & $40^{\circ} 29{ }^{\prime} 00^{\prime \prime} \mathrm{N}$ & $03^{\circ} 27^{\prime} 01 ” \mathrm{~W}$ & 213,17 & 8,74 \\
\hline Navacerrada & 1894 & $40^{\circ} 46^{\prime} 5 \mathrm{O}^{\prime \prime} \mathrm{N}$ & $04^{\circ} \mathrm{OO}^{\prime} 37^{\prime \prime} \mathrm{W}$ & 1114,56 & 11,63 \\
\hline Málaga (aeropuerto) & 7 & $36^{\circ} 40^{\prime} 00 ” \mathrm{~N}$ & $04^{\circ} 29 ' 17^{\prime \prime} \mathrm{W}$ & 310,60 & 9,17 \\
\hline Melilla & 47 & $35^{\circ} 16^{\prime} 40^{\prime \prime} \mathrm{N}$ & $\mathrm{O} 2^{\circ} 57^{\prime} 19 ” \mathrm{~W}$ & 297,10 & 13,88 \\
\hline Murcia (Alcantarilla) & 85 & $37^{\circ} 57^{\prime} 28 ” \mathrm{~N}$ & $01^{\circ} 13^{\prime} 47^{\prime \prime} \mathrm{W}$ & 115,61 & 4,79 \\
\hline San Javier (aeropuerto) & 4 & $37^{\circ} 47^{\prime} 20 ” \mathrm{~N}$ & $\mathrm{OO}^{\circ} 48{ }^{\prime} 12^{\prime \prime} \mathrm{W}$ & 288,50 & 16,55 \\
\hline Pamplona (aeropuerto) & 459 & $42^{\circ} 46^{\prime} 37^{\prime \prime N}$ & 01 ${ }^{\circ} 399^{\prime} 00^{\prime \prime W}$ & 417,40 & 11,54 \\
\hline Pontevedra & 108 & $42^{\circ} 26^{\prime} 24^{\prime \prime} \mathrm{N}$ & $08^{\circ} 36$ '59"W & 689,21 & 4,57 \\
\hline Vigo (aeropuerto) & 261 & $42^{\circ} 14^{\prime} 22 ” \mathrm{~N}$ & $08^{\circ} 37^{\prime} 26^{\prime \prime} \mathrm{W}$ & 1274,87 & 9,80 \\
\hline Salamanca (aeropuerto) & 790 & $40^{\circ} 57^{\prime} 34^{\prime \prime} \mathrm{N}$ & $05^{\circ} 294^{\prime \prime} \mathrm{W}$ & 320,54 & 17,25 \\
\hline
\end{tabular}


Tabla 1. Parámetros de solicitación obtenidos en los 80 emplazamientos analizados (continuación).

\begin{tabular}{|c|c|c|c|c|c|}
\hline EMPLAZAMIENTO & $\begin{array}{c}\text { Altitud } \\
\text { (m) }\end{array}$ & $\begin{array}{l}\text { Latitud } \\
\text { (DMS) }\end{array}$ & $\begin{array}{l}\text { Longitud } \\
\text { (DMS) }\end{array}$ & $\begin{array}{c}I_{A} \\
\left(1 / m^{2} \mathbf{a n n o}\right)\end{array}$ & $\begin{array}{c}\boldsymbol{D R W P} \\
(\mathrm{Pa})\end{array}$ \\
\hline Salamanca & 775 & $40^{\circ} 57^{\prime} 23 ” \mathrm{~N}$ & $05^{\circ} 39^{\prime} 41^{\prime \prime} \mathrm{W}$ & 227,79 & 7,86 \\
\hline Segovia & 1005 & $40^{\circ} 56^{\prime} 52^{\prime \prime} \mathrm{N}$ & $04^{\circ} 07^{\prime} 38^{\prime \prime} \mathrm{W}$ & 240,82 & 7,09 \\
\hline Morón de la Frontera & 87 & $37^{\circ} 09^{\prime} 30 " \mathrm{~N}$ & $\mathrm{O}^{\circ} 36^{\prime} 57^{\prime \prime} \mathrm{W}$ & 515,93 & 18,94 \\
\hline Sevilla (aeropuerto) & 34 & $37^{\circ} 25^{\prime} 00 ” \mathrm{~N}$ & $\mathrm{O}^{\circ} 52^{\prime} 45^{\prime \prime} \mathrm{W}$ & 428,40 & 13,01 \\
\hline Soria & 1082 & $41^{\circ} 46^{\prime} 30^{\prime \prime} \mathrm{N}$ & $\mathrm{O}^{\circ} 28{ }^{\circ} 59^{\prime \prime} \mathrm{W}$ & 315,40 & 10,16 \\
\hline Hierro (aeropuerto) & 32 & $27^{\circ} 49^{\prime} 08^{\prime \prime N}$ & $17^{\circ} 53^{\prime} 20^{\prime \prime} \mathrm{W}$ & 170,82 & 23,35 \\
\hline Izaña & 2371 & $28^{\circ} 18^{\prime} 32^{\prime \prime} \mathrm{N}$ & $16^{\circ} 29^{\prime} 58^{\prime \prime} \mathrm{W}$ & 914,94 & 62,28 \\
\hline La Palma (aeropuerto) & 33 & $28^{\circ} 37^{\prime} 59^{\prime \prime} \mathrm{N}$ & $17^{\circ} 45^{\prime} 18^{\prime \prime} \mathrm{W}$ & 344,98 & 22,56 \\
\hline Sta. Cruz de Tenerife & 35 & $28^{\circ} 27^{\prime} 48^{\prime \prime} \mathrm{N}$ & $16^{\circ} 15^{\prime} 19^{\prime \prime} \mathrm{W}$ & 134,35 & 8,31 \\
\hline Tenerife Norte (aeropuerto) & 632 & $28^{\circ} 28^{\prime} 39^{\prime \prime} \mathrm{N}$ & $16^{\circ} 19^{\prime} 46^{\prime \prime W}$ & 600,14 & 24,11 \\
\hline Tenerife Sur (aeropuerto) & 64 & $28^{\circ} \mathrm{O} 2^{\prime} 51^{\prime \prime} \mathrm{N}$ & $16^{\circ} 33^{\prime} 39^{\prime \prime W}$ & 121,57 & 22,86 \\
\hline Reus (aeropuerto) & 71 & $41^{\circ} 08^{\prime} 59^{\prime \prime} \mathrm{N}$ & $01^{\circ} 10^{\prime} 44^{\prime \prime} \mathrm{E}$ & 276,98 & 9,07 \\
\hline Tortosa & 44 & $40^{\circ} 49^{\prime} 14^{\prime \prime} \mathrm{N}$ & $\mathrm{OO}^{\circ} 29^{\prime} 29^{\prime \prime} \mathrm{E}$ & 216,69 & 5,67 \\
\hline Toledo & 515 & $39^{\circ} 53^{\prime} 05^{\prime \prime} \mathrm{N}$ & $04^{\circ} \mathrm{O} 2^{\prime} 58^{\prime \prime} \mathrm{W}$ & 193,24 & 8,69 \\
\hline Valencia (aeropuerto) & 69 & $39^{\circ} 29^{\prime} 12^{\prime \prime} \mathrm{N}$ & $\mathrm{OO}^{\circ} 28^{\prime} 24^{\prime \prime} \mathrm{W}$ & 267,21 & 10,77 \\
\hline Valencia & 11 & $39^{\circ} 28^{\prime} 50^{\prime \prime} \mathrm{N}$ & $\mathrm{OO}^{\circ} 21^{\prime} 59^{\prime \prime} \mathrm{W}$ & 176,81 & 3,94 \\
\hline Valladolid (Villanubla) & 846 & $41^{\circ} 42^{\prime} 00 ” \mathrm{~N}$ & $\mathrm{O}^{\circ} 51^{\prime} \mathrm{OO} ” \mathrm{~W}$ & 405,33 & 18,80 \\
\hline Valladolid & 735 & $41^{\circ} 39^{\prime} 00 ” \mathrm{~N}$ & $\mathrm{O}^{\circ} 46^{\prime} \mathrm{OO}$ ” W & 209,67 & 6,64 \\
\hline Bilbao (aeropuerto) & 42 & $43^{\circ} 17^{\prime} 53^{\prime \prime} \mathrm{N}$ & $\mathrm{O} 2^{\circ} 54^{\prime} 21^{\prime \prime} \mathrm{W}$ & 843,36 & 12,47 \\
\hline Zamora & 656 & $41^{\circ} 31^{\prime} \mathrm{OO}=\mathrm{N}$ & $05^{\circ} 44^{\prime} \mathrm{O} 1^{\prime \prime W}$ & 209,60 & 6,74 \\
\hline Daroca & 779 & $41^{\circ} 06^{\prime} 53^{\prime \prime} \mathrm{N}$ & $\mathrm{O}^{\circ} 24^{\prime} 39^{\prime \prime} \mathrm{W}$ & 124,14 & 3,28 \\
\hline Zaragoza (aeropuerto) & 247 & $41^{\circ} 39^{\prime} 43^{\prime \prime} \mathrm{N}$ & $\mathrm{O}^{\circ} \mathrm{OO} 29 \mathrm{O}^{\prime \prime} \mathrm{W}$ & 217,57 & 15,69 \\
\hline
\end{tabular}

Los mapas de exposición anual aportados en este trabajo (Figuras 5 y 6), junto con los aportados por trabajos precedentes y referidos a periodos de humedecimiento (20) (30), hacen posible un análisis completo de la solicitación por humedad, que podría ser utilizado como punto de partida para una hipotética revisión y mejora futura del Documento Básico.

La utilización conjunta de los índices de exposición recogidos en estos mapas para definir el grado de impermeabilidad requerido a las fachadas, permitiría resolver los puntos de mejora identificados en el apartado 2. Todo ello redundaría en una mejor asignación de las soluciones constructivas necesarias para hacer frente a la exigencia por humedad realmente presente sobre los cerramientos verticales, mejorando la prestacionalidad del DB-HS1. El desarrollo de esta línea de trabajo, mediante el análisis de un mayor número de emplazamientos en todo el país, así como la caracterización direccional de ambas exposiciones, permitiría igualmente una caracterización más completa y adecuada del territorio.

\section{CONCLUSIONES}

Este trabajo ha puesto de manifiesto las posibilidades de mejora actualmente existentes en el Código Técnico de la Edificación DB-HS1 para determinar la solicitación por humedad sobre los cerramientos verticales de edificación. Igualmente, se han examinado los parámetros utilizados in- ternacionalmente para caracterizar los dos factores más relevantes en esta solicitación: el aporte de agua recibido por los cerramientos verticales y la presión eólica simultánea a dicho aporte de agua. Estos parámetros han sido determinados en un número representativo de emplazamientos españoles, utilizando procedimientos de cálculo estandarizados en el ámbito internacional y adaptados a la naturaleza de los registros climáticos disponibles en España.

Los resultados obtenidos y los mapas elaborados en base a ellos, podrían ser tenidos en cuenta en futuras revisiones del DB-HS1 para la mejora prestacional del grado de impermeabilidad asignado para los cerramientos verticales de edificación.

\section{AGRADECIMIENTOS}

Resultados obtenidos mediante la información cedida por la Agencia Estatal de Meteorología, Ministerio de Medio Ambiente y Medio Rural y Marino. Esta investigación ha sido parcialmente financiada por el Ministerio de Ciencia e Innovación y cofinanciada con fondos FEDER a través del proyecto de investigación BIA2012-31609. Se quiere reconocer la labor de D. Javier Escuer Gracia en la elaboración de los mapas presentados, así como la colaboración del grupo de investigación GICONSIME, perteneciente a la Universidad de Oviedo.

\section{REFERENCIAS}

(1) Broto, C. (2006). Enciclopedia Broto de las patologías de la construcción. Barcelona: Links Barcelona, S.A.

(2) Lozano, A., Lozano, G., del Coz, J.J. (2006). Curso: Síndrome, patología y terapéutica de las humedades. Gijón: Autoeditor.

(3) Sanders, C. (1996). Heat, air and moisture transfer in insulated envelope parts: Environmental conditions. Final report, Annex 24, Vol. 2. Leuven: International Energy Agency. 
(4) Del Coz, J.J., Rabanal, F.P., García, P.J., Domínguez, J., Rodríguez, B., Pérez, J.M. (2013). Hygrothermal properties of lightweight concrete: Experiments and numerical fitting study. Construction and Building Materials, 40:543-555, doi: http://dx.doi.org/10.1016/j.conbuildmat.2012.11.045.

(5) Bornehag, C.G., Blomquist, G., Gyntelberg, F., Järvholm, B., Malmberg, P., Nordvall, L., Nielsen, A., Pershagen, G., Sundell, J. (2001). Dampness in buildings and health. Indoor Air, 11(2): 72-86, doi: http://dx.doi.org/10.1034/j.16000668.2001.110202.x.

(6) World Health Organization. (2011). Environmental burden of disease associated with inadequate housing. Methods for quantifying health impacts of selected housing risks in the WHO European Region. Copenhagen: World Health Organization.

(7) Ministerio de Vivienda. (2006). Código Técnico de la Edificación. Documento Básico - Salubridad. Madrid: Ministerio de Vivienda.

(8) Blocken, B., Carmeliet, J. (2004) A review of wind-driven rain research in building science. Journal of Wind Engineering and Industrial Aerodynamics, 92(13): 1079-1130, doi: http://dx.doi.org/10.1016/j.jweia.2004.06.003.

(9) Cornick, S.M., Lacasse, M.A. (2005). A review of climate loads relevant to assessing the watertightness performance of walls, windows, and wall-window interfaces. Journal of ASTM International, 2(10): 1-16, doi: http://dx.doi.org/10.1520/JAI12505.

(10) Hoppestad, S. (1955). Slagregn i Norge (Lluvia batiente en Noruega, en noruego). Report no. 13. Oslo: Norwegian Building Research Institute.

(11) Lacy, R.E. (1965). Driving-rain maps and the onslaught of rain on buildings. En Proceedings of RILEM/CIB symposium on moisture problems in buildings. Helsinki.

(12) Blocken, B., Carmeliet, J. (2008). Guidelines for the required time resolution of meteorological input data for winddriven rain calculations on buildings. Journal of Wind Engineering and Industrial Aerodynamics, 96(5): 621-639, doi: http://dx.doi.org/10.1016/j.jweia.2008.02.008.

(13) Blocken, B., Carmeliet, J. (2007). On the error associated with the use of hourly data in wind-driven rain calculations on building facades. Atmospheric Environment, 41(11): 2335-2343, doi: http://dx.doi.org/10.1016/j.atmosenv.2006.11.014.

(14) Lacy, R.E. (1977). Climate and Building in Britain. London: Her Majesty's Stationery Office.

(15) Henriques, F.M.A. (1992). Quantification of wind-driven rain. An experimental approach. A general review on driven rain and details of an experiment in Portugal to supplement existing research results in Norway, the UK and elsewhere. Building Research and Information, 20(5): 295-297, doi: http://dx.doi.org/10.1080/09613219208727227.

(16) Straube, J.F., Burnett, E.F.P. (2000). Simplified Prediction of Driving Rain Deposition. En Proceedings of International Building Physics Conference, Eindhoven, (pp. 375-382). Eindhoven.

(17) Lacy, R.E., Shellard, H.C. (1962). An index of driving rain. The Meteorological Magazine, 91(1080): 177-84.

(18) Sahal, N. (2006). Proposed approach for defining climate regions for Turkey based on annual driving rain index and heating degree-days for building envelope design. Building and Environment, 41(4): 520-526, doi: http://dx.doi. org/10.1016/j.buildenv.2005.07.004.

(19) Giarma, C., Aravantinos, D. (2011). Estimation of building components' exposure to moisture in Greece based on wind, rainfall and other climatic data. Journal of Wind Engineering and Industrial Aerodynamics, 99(2-3): 91-102, doi: http://dx.doi.org/10.1016/j.jweia.2010.12.001.

(20) Pérez, J.M., Domínguez, J., Rodríguez, B., del Coz, J.J., Cano, E. (2012). Estimation of the exposure to moisture in Spain from daily wind and rain data. Building and Environment, 57: 259-270, doi: http://dx.doi.org/10.1016/j.buildenv.2012.05.010.

(21) CEN. (2009). EN ISO 15927-3 Hygrothermal performance of buildings - Calculation and presentation of climatic data Part 3: Calculation of a driving rain index for vertical surfaces from hourly wind and rain data. European Committee for Standardization.

(22) AENOR. (2011). UNE-EN ISO 15927-3 Comportamiento higrotérmico de los edificios - Cálculo y presentación de datos climáticos. Parte 3: Cálculo de un índice de lluvia para superficies verticales a partir de datos horarios de viento y lluvia. Madrid: Asociación Española de Normalización y Certificación.

(23) Gumbel, E.J. (1958). Statistcs of extremes. New York: Columbia University Press.

(24) Pérez, J.M., Domínguez, J., Rodríguez, B., del Coz, J. J., Suñén, E. (2013). Optimised method for estimating directional driving rain from synoptic observation data. Journal of Wind Engineering and Industrial Aerodynamics, 113: 1-11, doi: http://dx.doi.org/10.1016/j.jweia.2012.12.001.

(25) CEN. (2001). EN 12865:2001. Hygrothermal performance of building components and building elements. Determination of the resistance of external wall systems to driving rain under pulsating air pressure. European Committee for Standardization.

(26) ASTM. (2009). ASTM E331-oo. Standard test method for water penetration of exterior windows, skylights, doors, and curtain walls by uniform static air pressure difference. American Society for Testing and Materials.

(27) Welsh, R.E., Skinner, W.R., Morris, R.J. (1989). A climatology of driving rain pressure for Canada. Climate and Atmospheric Research Directorate Draft Report. Canada: Environment Canada, Atmospheric Environment Service.

(28) Bsi. (1992). BS 8104 Code of practice for assessing exposure of walls to wind-driven rain. British Standards Institution.

(29) Office of the Deputy Prime Minister. (2010). Approved Document C. Site preparation and resistance to contaminants and moisture. London: The Building Regulations.

(30) Pérez, J.M., Domínguez, J., Rodríguez, B., del Coz, J.J., Cano, E. (2013). Combined use of wind-driven rain and wind pressure to define water penetration risk into building façades: the Spanish case. Building and Environment, 64:46-56, doi: http://dx.doi.org/10.1016/j.buildenv.2013.03.004. 